Open Access

\title{
The Salford Lung Study protocol: a pragmatic, randomised phase III real-world effectiveness trial in chronic obstructive pulmonary disease
}

\author{
Nawar Diar Bakerly', Ashley Woodcock², John P. New', J. Martin Gibson', Wei Wu'3 , David Leather ${ }^{4}$ \\ and Jørgen Vestbo ${ }^{2,5^{*}}$ (iD
}

\begin{abstract}
Background: New treatments need to be evaluated in real-world clinical practice to account for co-morbidities, adherence and polypharmacy.

Methods: Patients with chronic obstructive pulmonary disease (COPD), $\geq 40$ years old, with exacerbation in the previous 3 years are randomised 1:1 to once-daily fluticasone furoate $100 \mu \mathrm{g} / \mathrm{vilanterol} 25 \mathrm{\mu g}$ in a novel dry-powder inhaler versus continuing their existing therapy. The primary endpoint is the mean annual rate of COPD exacerbations; an electronic medical record allows real-time collection and monitoring of endpoint and safety data.
\end{abstract}

Conclusions: The Salford Lung Study is the world's first pragmatic randomised controlled trial of a pre-licensed medication in COPD.

Trial registration: Clinicaltrials.gov identifier NCT01551758.

\section{Introduction}

Double-blind randomised controlled trials (RCTs) in chronic obstructive pulmonary disease (COPD) have indicated that inhaled corticosteroids (ICS) combined with a long-acting $\beta_{2}$-agonist (LABA) are more effective than the individual components in managing stable COPD, reducing exacerbations and improving lung function and health status [1]. However, double-blind RCTs differ from real life in having highly selective eligibility criteria, and enrolling participants who are not representative of patients in clinical practice and have much higher adherence [2]. The once-daily combination of the ICS fluticasone furoate (FF) and the novel LABA vilanterol (VI) $\left(\right.$ Relvar $\left.^{\circ}\right)$ in a patient-friendly dry-powder inhaler (DPI) (Ellipta ${ }^{\circ}$ ) has the potential for improved adherence over the currently available twice-daily

\footnotetext{
* Correspondence: jorgen.vestbo@manchester.ac.uk

${ }^{2}$ Institute of Inflammation and Repair, Manchester Academic Health Science Centre, University of Manchester, Manchester, UK

${ }^{5}$ Centre for Respiratory Medicine and Allergy, 2nd Floor Education and Research Centre, University Hospital of South Manchester NHS Foundation Trust, Manchester M23 9LT, UK

Full list of author information is available at the end of the article
}

ICS/LABA combinations, with improved clinical effectiveness in a real-world setting [3].

The Salford Lung Study (SLS) is the world's first pragmatic RCT (pRCT) of an investigational medication. SLS will evaluate the effectiveness and safety of the FF/VI combination compared with existing maintenance therapy in a large, real-world population of patients with COPD in conditions of normal care. The study is being conducted in and around Salford, UK. Salford has a high prevalence of COPD in a community served by a single hospital and an established electronic medical record (EMR), connecting both primary and secondary care. Pharmacies also collaborate to allow patients to collect study medication from their usual community pharmacy.

\section{Methods \\ Study design}

SLS is a 12-month, open-label, phase III pRCT, evaluating the effectiveness and safety of FF/VI (Relvar; $100 \mu \mathrm{g} / 25 \mu \mathrm{g}$ once daily, delivered by a novel DPI, Ellipta ${ }^{\circ}$ ) in patients with COPD (clinicaltrials.gov identifier NCT01551758). The study is conducted in accordance with the International Conference on 
Harmonisation, Good Clinical Practice (GCP), all applicable data protection requirements and the ethical principles outlined in the Declaration of Helsinki 2008. The study was approved by the Ethics committee, National Research Ethics Service Committee North West, Greater Manchester South.

\section{Patients}

All patients with COPD at 66 primary care sites (at the time of manuscript preparation) in and around Salford and South Manchester are identified by their general practitioner (GP) from practice databases and invited to participate in the study.

Eligibility criteria include:

- aged $\geq 40$ years

- documented GP diagnosis of COPD

- regular maintenance inhaler therapy (ICS alone or in combination with long-acting bronchodilator, one or more long-acting bronchodilators, or triple therapy [ICS/LABA plus long-acting muscarinic antagonist])

- at least one COPD exacerbation in the last 3 years.

Minimal exclusion criteria:

- an exacerbation within the previous 2 weeks

- chronic oral corticosteroid use.

At visit 1, patients are offered study participation through written informed consent (Fig. 1). At visit 2 (160 days after visit 1 ), patients are randomised (1:1) to receive either FF/VI or to continue their usual maintenance therapy. Patients randomised to FF/VI are instructed in the use of the Ellipta DPI. Patients randomised to their usual maintenance therapy are re-trained in the correct techniques and dosing. Baseline assessments are performed at visit 2 , including quality of life and disease characteristics (e.g., disease duration, COPD maintenance therapy, smoking status, lung function, medical history). If at months 3, 6 and 9 the patient has had no contact with their GP practice within the previous 8 weeks, the patient is contacted by telephone to assess any serious adverse events (SAEs) or non-serious adverse drug reactions (ADRs) (visits 3, 4 and 5). There is no additional intervention at these assessments. At 12 months, the final visit (6) is a face-to-face meeting with the patient at which the final assessment of outcomes is conducted.

\section{Participating sites \\ Primary care}

To preserve the real-world nature of the study, the patient experience is as close to routine care as possible. The study's principal investigators are the GPs. They are ideally placed to facilitate recruitment, identify and report SAEs or serious ADRs and report study endpoints. GPs may make treatment adjustments according to their clinical opinion. Repeat prescriptions of study medication are issued by GPs as usual, and collected by patients from their usual pharmacy. As very few participating GPs had experience of clinical trial participation, all GPs have received training and support in GCP, patient recruitment, study protocol, coding of healthcare issues and general research procedures.

\section{Pharmacy}

Every pharmacy in Salford and others in South Manchester agreed to participate in the study. As with GPs, very few pharmacists had experience of clinical trial participation. All staff $(>500)$ at participating pharmacies have received training in GCP and safety reporting and standard operating procedures were established. Initially, pharmacies faxed copies of study treatment prescriptions

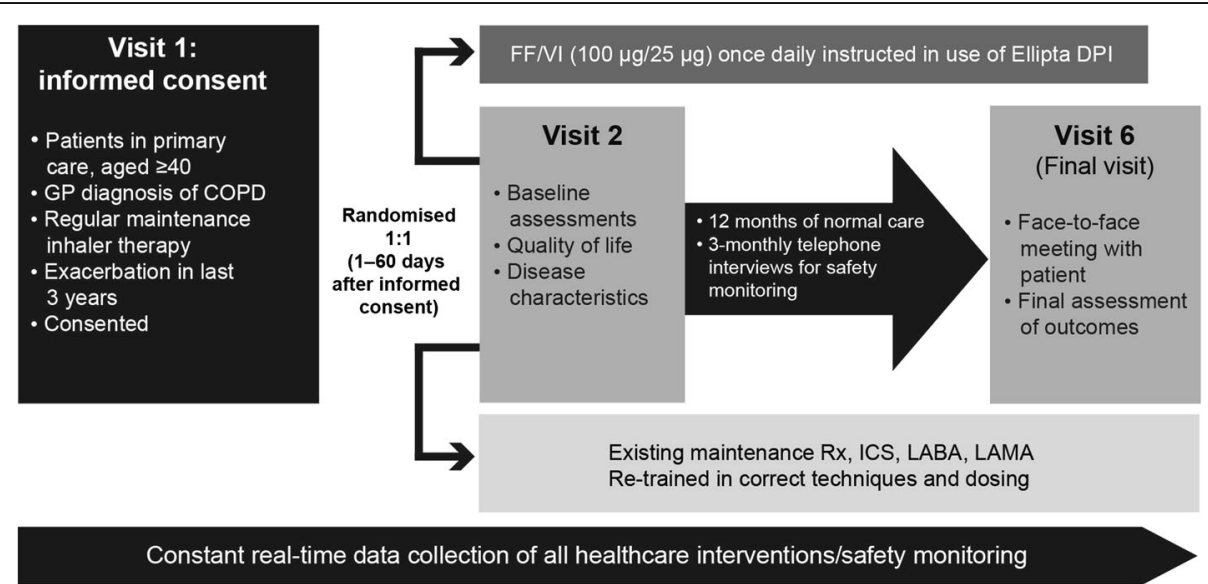

Fig. 1 Study design. COPD = chronic obstructive pulmonary disease; DPI = dry-powder inhaler; FF = fluticasone furoate; GP = general practitioner; ICS = inhaled corticosteroid; LABA = long-acting $\beta_{2}$-agonist; LAMA = long-acting muscarinic antagonist; $R x=$ treatment; $V I=$ vilanterol 
to the study coordination centre, but these are now collected electronically. Prescription collection data are used to assess treatment adherence.

\section{Hospital}

The majority of admissions are to the local hospitals: Salford Royal Hospital and University Hospital of South Manchester. Admissions are identified electronically and assessed by a separate secondary care team within $48 \mathrm{~h}$.

\section{Data monitoring}

All hospital admissions, outpatient and emergency department visits are identified from the EMR database (whenever and wherever they occur). From primary care, all healthcare contacts, out-of-hours activity and prescriptions of antibiotics or oral steroids can be identified. These events are reviewed by the study research team and classified as COPD or non-COPD related. Furthermore, the EMRs capture suspected unexpected serious adverse reactions (e.g., reduced kidney function or elevated liver function tests) and, for the purposes of SLS, include data from external sources to identify, e.g., deaths or National Health Service (NHS) hospital admissions outside Salford. NorthWest EHealth (www.nweh.org.uk) manages the EMRs, enabling data on study endpoints and patient safety to be collected continuously and remotely in near-real time, without the need for face-to-face patient contact.

\section{Endpoints}

\section{Effectiveness}

The primary endpoint is the mean annual rate of moderate or severe exacerbations. Secondary endpoints include time to first exacerbation and healthcare utilisation. Other endpoints include hospitalisations, use of rescue medication, the COPD Assessment Test (CAT) [4] and EuroQoL-5 dimensions (EQ-5D) questionnaire, listed and defined in Table 1. EMR data for effectiveness endpoints are independently verified by the research team (GP, research nurse, research doctor).

\section{Safety}

Safety endpoints include death, pneumonia, frequency and type of SAEs, and ADRs. Investigators and site staff are responsible for detecting, documenting and reporting SAEs and ADRs on electronic case report forms (eCRFs), which are continuously monitored by a dedicated clinical safety team.

\section{Withdrawals}

Patients with COPD exacerbation during the treatment period may remain in the study and continue to take study medication at the discretion of their GP. Severe COPD exacerbations are reported as SAEs. Patients with
Table 1 Study endpoints

\begin{tabular}{ll}
\hline Endpoint & Definitions \\
\hline Primary endpoint & \\
Mean annual rate of moderate & - Moderate exacerbation: patient \\
or severe exacerbations & receiving an exacerbation-related \\
& prescription of oral corticosteroids \\
& and/or antibiotic (with or without \\
& NHS contact) not requiring \\
& hospitalisation \\
& Severe exacerbation: an \\
& exacerbation-related \\
& hospitalisation
\end{tabular}

Secondary endpoints

- COPD-related secondary care contacts

- COPD-related primary care contacts

- All secondary care contacts

- All primary care contacts

- Time to discontinuation of initial therapy

- Time to addition of a further COPD controller medication

- Time to first moderate/severe exacerbation

- Time to first severe exacerbation (i.e., hospitalisation)

Other endpoints

- Number of hospitalisations

- Number of days in hospital

- Total number of respiratoryrelated home visits (including out-of-hours calls) and telephone consultations

- CAT: disease management, quality of life

- EQ-5D

- Adherence to study medication

- Number of salbutamol inhalers collected by the patients from study-enrolled community pharmacies over the 12-month treatment period

CAT COPD Assessment Test, COPD chronic obstructive pulmonary disease, EQ-5D EuroQol Questionnaire, MARS-A Medication Adherence Report Scale for Asthma, NHS National Health Service

worsening COPD status while on study treatment may have their medication adjusted at the GP's discretion or receive other permitted COPD therapies. If, in the investigator's opinion, the frequency or severity of exacerbations prevents ongoing participation, the patient can be withdrawn. The reason for withdrawal is recorded in the eCRF and patients are followed up for 12 months following randomisation, with their consent. 


\section{Statistical analysis and rationale}

The primary efficacy analysis population is intent-totreat, defined as all randomised patients who have received at least one prescription of study medication.

Sample size calculations are based on the primary endpoint (mean annual rate of moderate and severe exacerbations) and primary efficacy analysis population. A total of 2238 patients (1119 patients per treatment group) are needed. The study has $80 \%$ power to detect a relative reduction of $12 \%$ in the mean annual moderate or severe exacerbation rate, assuming a mean exacerbation rate of 2.3 for the control group [5]. Calculations are based on a negative binomial regression with a dispersion rate of 0.7 and use a two-sided $5 \%$ significance level.

To account for variation in treatment response between patient subgroups, randomisation is stratified by baseline maintenance therapy and by history of COPD exacerbation in the previous 12 months (yes/no) to ensure treatment balance for the primary efficacy analysis population. Analyses based on subgroups are also planned; subgroups will be defined on baseline medication, lung function, comorbidities and other factors.

\section{Discussion}

SLS is a unique pRCT and, to our knowledge, the first prospective real-world comparative effectiveness study of an investigational medicine, which commenced in March 2012, prior to UK regulatory approval (launch date January 2014). The pragmatic inclusion criteria in SLS represent the broad definition of a patient eligible for COPD maintenance therapy in the real world, irrespective of co-morbidities. Study accessibility is maximised by employing minimal exclusion criteria and requirements for additional GP visits. Medicine prescription and supply is achieved as usual, through the patient's own GP and pharmacy.

Real-world outcomes can be assessed by observational studies that provide high external validity but in contrast have low internal validity [6]. With the limitations in observational studies and those in double-blind RCTs [2] such studies alone may not fully reflect the true impact and value of treatments for COPD. As such, welldesigned pRCTs may offer complementary data to these standard types of studies, representing true real-world effectiveness.

Performing a study of a pre-licence drug in a realworld setting has posed many new challenges in study design, operational planning and study support. Patient safety is a priority in studying a pre-licence medicine. Patient safety in SLS is monitored in almost real-time by a combination of remote surveillance of EMRs and clinical monitoring. This sets a new standard, in which safety signals can be seen more quickly than in conventional RCTs. The major challenge has been managing and assessing the relevance and importance of safety signals within the huge volume of healthcare data being generated.

The SLS has limitations. Patients are not blinded and although patients are far less selected than in a usual efficacy trial, some selection bias cannot be precluded. Also, the fact that patients are recruited on the basis of a diagnosis from an electronic medical record and not from a specialist clinic could raise concerns. However, our approach mirrors the real world and a recent study found that registered data had satisfactory validity [7].

\section{Conclusions}

SLS is an innovative project with the aim of evaluating the safety and effectiveness of an investigational medicine in a real-world setting. Data from SLS will allow a better understanding of the risk/benefit profile of the FF/VI combination in the wider COPD community. The study will likely be a role model for future evaluation of effectiveness of new therapies.

\begin{abstract}
Abbreviations
ADR: Adverse drug reaction; CAT: COPD Assessment Test; COPD: Chronic obstructive pulmonary disease; DPI: Dry-powder inhaler; eCRF: Electronic case report form; EMR: Electronic medical record; EQ-5D: EuroQoL-5 dimensions (EQ-5D) questionnaire; FF: Fluticasone furoate; GCP: Good Clinical Practice; GP: General practitioner; ICS: Inhaled corticosteroid; LABA: Long-acting $\beta_{2}$-agonist; LAMA: Long-acting muscarinic antagonist; MARS-A: Medication Adherence Report Scale for Asthma; NHS: National Health Service; pRCT: Pragmatic randomised controlled trial; RCT: Randomised controlled trial; Rx: Treatment; SAE: Serious adverse event; SLS: Salford Lung Study; VI: Vilanterol.
\end{abstract}

\section{Competing interests}

NDB's employing organisation provides IT support to GlaxoSmithKline. He has received educational grants and speaker's fees from GlaxoSmithKline and Novartis, and support for attending educational conferences from Boehringer Ingelheim, GlaxoSmithKline and Novartis. AW has acted on advisory boards and provided consultancy for Almirall, Chiesi, Cytos and GlaxoSmithKline. He has received travel support to speak at international meetings from Boehringer Ingelheim and GlaxoSmithKline. He is an investigator on cough and asthma studies for Afferent and GlaxoSmithKline. JPN has received consulting and speaker's fees, and an educational grant from GlaxoSmithKline. JMG's institution has received funding from GlaxoSmithKline as the SLS study sponsor. WW is an employee of, and holds shares/stock options in, GlaxoSmithKline. DL is an employee of, and holds shares/stock options in, GlaxoSmithKline. JV has received travel support and consultancy fees from GlaxoSmithKline (related to the SLS study); in addition, he has received consultancy fees from Almirall, AstraZeneca, Bioxydyn, Boehringer-Ingelheim, Chiesi, GlaxoSmithKline (outside the SLS study), Novartis, Syntaxin and Takeda (Nycomed), and speaker's fees from AstraZeneca, Boehringer Ingelheim, Chiesi, GlaxoSmithKline, Novartis and Takeda (Nycomed). His wife has previously worked for AstraZeneca, Ferring and GlaxoSmithKline (until 2009).

\section{Authors' contributions}

All authors are involved in the design and implementation of the Salford Lung Study and contributed equally to the preparation of this paper, including development of the outline, review of all drafts, final approval and decision to submit the manuscript to Respiratory Research.

\section{Acknowledgements}

The authors thank Kerry Acheson of iMed Comms, Macclesfield, UK, who provided medical writing support, which was funded by GlaxoSmithKline. 


\section{Funding support}

GlaxoSmithKline are sponsors of the Salford Lung Study and provided scientific support for the study design, protocol writing, data collection, analysis, interpretation of study data, and funded medical writing services.

\section{Author details}

'Salford Royal NHS Foundation Trust, Salford, UK. ${ }^{2}$ Institute of Inflammation and Repair, Manchester Academic Health Science Centre, University of Manchester, Manchester, UK. ${ }^{3}$ GlaxoSmithKline, Research Triangle Park, Durham, NC, USA. ${ }^{4}$ GSK Respiratory Centre of Excellence, GlaxoSmithKline UK Ltd, Uxbridge, UK. ${ }^{5}$ Centre for Respiratory Medicine and Allergy, 2nd Floor Education and Research Centre, University Hospital of South Manchester NHS Foundation Trust, Manchester M23 9LT, UK.

Received: 2 July 2015 Accepted: 26 August 2015

Published online: 04 September 2015

\section{References}

1. Calverley PM, Rennard SI. What have we learned from large drug treatment trials in COPD? Lancet. 2007;370:774-85.

2. Roche N, Reddel H, Martin R, Brusselle G, Papi A, Thomas M, et al. Quality standards for real-world research. Focus on observational database studies of comparative effectiveness. Ann Am Thorac Soc. 2014;11 Suppl 2:S99-104.

3. Claxton AJ, Cramer J, Pierce C. A systematic review of the associations between dose regimens and medication compliance. Clin Ther. 2001;23:1296-310.

4. Jones P, Harding G, Wiklund I, Berry P, Leidy N. Improving the process and outcome of care in COPD: development of a standardised assessment tool. Prim Care Respir J. 2009;18:208-15.

5. New JP, Delderfield MR, Stein ND, Austin S, Vestbo J, Woodcock AA. Assessing the burden of asthma and COPD in Salford UK: retrospective analysis using a whole population electronic medical record. Eur Respir J. 2011;38 Suppl 55:732S. Abstract 4012.

6. Price D, Chisholm A, van der Molen T, Roche N, Hillyer EV, Bousquet J. Reassessing the evidence hierarchy in asthma: evaluating comparative effectiveness. Curr Allergy Asthma Rep. 2011;11:526-38.

7. Elkhenini HF, Davis KJ, Stein ND, New JP, Delderfield MR, Gibson M, et al. Using an electronic medical record (EMR) to conduct clinical trials: Salford Lung Study feasibility. BMC Med Inform Dec Making. 2015;15:8. doi:10.1186/s12911-015-0132-z.

\section{Submit your next manuscript to BioMed Central and take full advantage of:}

- Convenient online submission

- Thorough peer review

- No space constraints or color figure charges

- Immediate publication on acceptance

- Inclusion in PubMed, CAS, Scopus and Google Scholar

- Research which is freely available for redistribution 